\title{
Highly Regio-, Diastereo- and Enantio-selective Pd-Catalyzed Allylic Alkylation of Acyclic Ketone Enolates with Monosubstituted Allyl Substrates
}

Wen-Hua Zheng, Bao-Hui Zheng, Yan Zhang, Xue-long Hou*

State key Laboratory of Organometallic Chemistry, Shanghai Institute of Organic Chemistry, Chinese Academy of Sciences, 354 Fenglin Lu, Shanghai 200032, China

E-mail: xlhou@mail.sioc.ac.cn

General methods: Unless stated otherwise, all reactions were carried out in flame-dried glassware under a dry argon atmosphere. All solvents were purified and dried according to standard methods prior to use.

${ }^{1} \mathrm{H}$ and ${ }^{13} \mathrm{C}$ NMR spectra were recorded on a NMR instrument $(300 \mathrm{MHz}$ and $75 \mathrm{MHz}$, respectively). Data for ${ }^{1} \mathrm{H}$ NMR are recorded as follows: chemical shift $(\delta, \mathrm{ppm})$, multiplicity $(\mathrm{s}=$ singlet, $\mathrm{d}=$ doublet, $\mathrm{t}=$ triplet, $\mathrm{m}=$ multiplet or unresolved, $\mathrm{br}=$ broad singlet, coupling constant(s) in Hz, integration). Data for ${ }^{13} \mathrm{C}$ NMR are reported in terms of chemical shift ( $\delta$, ppm). Ligand $\left(R_{\text {phos }}, R\right)$-L6 was synthesized using our previously reported procedures [Hou, X.-L.; Sun, N. Org. Lett. 2004, 6, 4399.]

General Procedure for the Pd Catalyzed Asymmetric Allylic Alkylation:

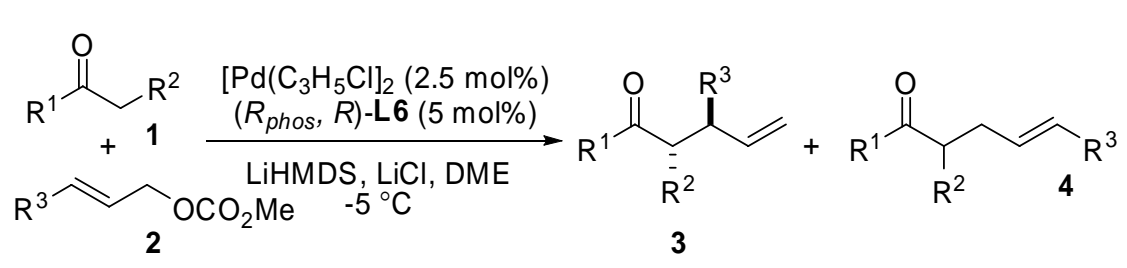

2
2a. $\mathrm{R}^{3}=\mathrm{Ph}$;
2b. $\mathrm{R}^{3}=p-\mathrm{FC}_{6} \mathrm{H}_{4}$;
2c. $\mathrm{R}^{3}=p-\mathrm{MeC}_{6} \mathrm{H}_{4}$
2d. $\mathrm{R}^{3}=m-\mathrm{BrC}_{6} \mathrm{H}_{4}$;
2e. $\mathrm{R}^{3}=p-\mathrm{MeOC}_{6} \mathrm{H}_{4}$;
2f. $\mathrm{R}^{3}=1$-Naphthyl;

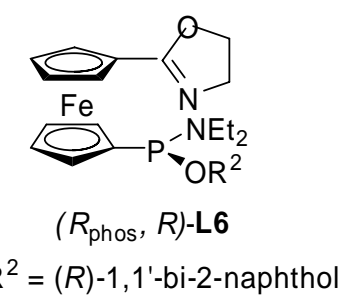

A dry Schlenk tube containing $\mathrm{LiCl}(0.25 \mathrm{mmol})$ was flame dried and flushed with Argon. 
Ketone $(0.25 \mathrm{mmol})$ and DME $(2.0 \mathrm{~mL})$ were added. LiHMDS $(1.0 \mathrm{M}$ in THF, $0.25 \mathrm{~mL}, 0.25$ mmol) was added drop wise with stirred for $30 \mathrm{~min}$. In an separate flushed flask, $\left[\mathrm{Pd}\left(\mathrm{C}_{3} \mathrm{H}_{5}\right) \mathrm{Cl}\right]_{2}$ $(1.8 \mathrm{mg}, 0.005 \mathrm{mmol})$ and ligand $\left(R_{\text {phos }}, R\right)-\mathbf{L} 6(0.01 \mathrm{mmol})$ were added followed by addition of DME $(1.0 \mathrm{~mL})$ with stirring at room temperature for $30 \mathrm{~min}$. The catalyst and enolate solution were cooled to specified temperature, and the catalyst added to the enolate solution. The allylic carbonate $(0.275 \mathrm{mmol})$ was then added and the resulted mixture was stirred for c.a. two hours (TLC control). The reaction mixture was then quenched by $\mathrm{H}_{2} \mathrm{O}(5 \mathrm{~mL})$ and partitioned between diethyl ether and $\mathrm{H}_{2} \mathrm{O}$. The aqueous phase was washed with diethyl ether $(3 \times 10 \mathrm{~mL})$ and the organic layer was combined, dried $\left(\mathrm{Na}_{2} \mathrm{SO}_{4}\right)$, filtered and concentrated in vacuo to afford a crude oil. Purification with preparative TLC furnished the product as colorless oil.

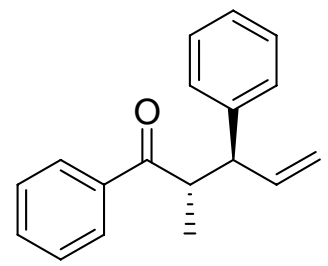

\section{2-Methyl-1,3-diphenyl-pent-4-en-1-one (3a)}

Yield: 83\%; B/L: >98/2; anti:syn: 9:1; ee (anti-3a): 98\%.

$[\alpha]_{\mathrm{D}}{ }^{25}=-56.9^{\circ}\left(1.41, \mathrm{CHCl}_{3}\right) ;{ }^{1} \mathrm{H} \mathrm{NMR}\left(300 \mathrm{MHz}, \mathrm{CDCl}_{3}\right)$ (taken as a mixture of diastereomers): $\delta$ (major diastereomer) $1.27(\mathrm{~d}, J=6.9 \mathrm{~Hz}, 1 \mathrm{H}), 3.74-3.80(\mathrm{~m}, 1 \mathrm{H}), 3.91-3.97(\mathrm{~m}, 1 \mathrm{H}), 5.11-5.17$ $(\mathrm{m}, 2 \mathrm{H})$, 5.97-6.08 (m, 1H), 7.07-7.52 (m, 8H), 7.79-7.82 (m, 2H); (minor diastereomer ) $0.99(\mathrm{~d}$, $J=6.9 \mathrm{~Hz}, 3 \mathrm{H}), 4.90-4.99(\mathrm{~m}, 2 \mathrm{H}) ;{ }^{13} \mathrm{C}$ NMR $\left(75 \mathrm{MHz}, \mathrm{CDCl}_{3}\right): \delta$ (major diastereomer) 203.4, $142.8,139.0,137.0,132.7,128.4,128.4,128.0,127.6,126.3,116.6,53.0,45.2,16.5 ; \mathrm{MS}$ (EI) m/z (rel) 250 (M+, 1), 235 (9), 117 (59), 115 (28), 105 (100), 91 (17), 77 (43), 51 (20); IR (film) 3029 (w), 2976 (w), 1683(s), 1449 (m), 1210 (m), 973 (m), 701 (s); HRMS Calcd. for $\mathrm{C}_{18} \mathrm{H}_{18} \mathrm{O}$ : 250.1258; Found: 250.1257. HPLC (Chiralcel OJ-H, hexane/i-Propanol $=90 / 10,0.7 \mathrm{~mL} / \mathrm{min}$, $254 \mathrm{~nm}) \mathrm{t}_{\mathrm{R}}=11.01 \mathrm{~min}, 14.16 \mathrm{~min}$. 
<smiles>C=C[C@H](c1ccc(F)cc1)[C@@H](C)C(=O)c1ccccc1</smiles>

\section{2-Methyl-1-phenyl-3-(p-fluoro-phenyl)-pent-4-en-1-one (3b)}

Yield: 90\%; B/L: >98/2; anti:syn: 8:1; ee (anti-3b): 98\%.

$[\alpha]_{\mathrm{D}}{ }^{25}=-41.8^{\mathrm{o}}\left(1.41, \mathrm{CHCl}_{3}\right) ;{ }^{1} \mathrm{H} \mathrm{NMR}\left(300 \mathrm{MHz}, \mathrm{CDCl}_{3}\right)$ (taken as a mixture of diastereomers): $\delta$ (major diastereomer) $1.26(\mathrm{~d}, J=6.9 \mathrm{~Hz}, 3 \mathrm{H}), 3.71-3.77(\mathrm{~m}, 1 \mathrm{H}), 3.82-3.90(\mathrm{~m}, 1 \mathrm{H}), 5.11-5.15$ $(\mathrm{m}, 2 \mathrm{H}), 5.92-6.04(\mathrm{~m}, 1 \mathrm{H}), 6.83-7.80(\mathrm{~m}, 9 \mathrm{H}) ; \delta$ (minor diastereomer $) 0.97(\mathrm{~d}, J=6.6 \mathrm{~Hz}, 3 \mathrm{H})$, 4.91-4.97 (m, 2H); ${ }^{13} \mathrm{C}$ NMR (75 $\mathrm{MHz}, \mathrm{CDCl}_{3}$ ): $\delta$ (major diastereomer) 203.2, 138.8, 132.8, 129.0, 128.9, 128.4, 128.1, 127.8, 116.6, 115.2, 115.0, 52.2, 45.2, 16.6; MS (EI) m/z (rel) 253 (6), 135 (43), 115 (13), 109 (11), 106 (7), 105 (100), 77 (48), 51 (18); IR (film) 2978 (w), 2933 (w), 1683 (s), 1598 (m), 1509 9s), 1449 (m), 1223 (s), 973 (m), 832 (m), 708 (m); HRMS for $\mathrm{C}_{18} \mathrm{H}_{17} \mathrm{OF}$ : 268.1263; Found: 268.1267; HPLC (Chiralcel OJ-H, hexane/i-Propanol = 95/5, 0.5 $\mathrm{mL} / \mathrm{min}, 254 \mathrm{~nm}) \mathrm{t}_{\mathrm{R}}=13.52 \mathrm{~min}, 16.55 \mathrm{~min}$.

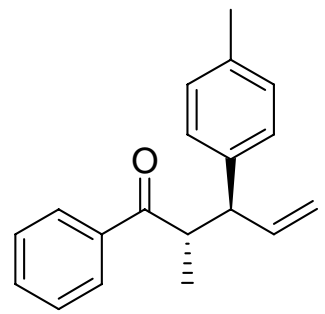

\section{2-Methyl-1-phenyl-3-(p-methyl-phenyl)-pent-4-en-1-one (3c)}

Yield: 80\%; B/L: >98/2; anti:syn: 9:1; ee (anti-3c): 96\%.

$[\alpha]_{\mathrm{D}}{ }^{25}=-52.4^{\mathrm{o}}\left(1.41, \mathrm{CHCl}_{3}\right) ;{ }^{1} \mathrm{H} \mathrm{NMR}\left(300 \mathrm{MHz}, \mathrm{CDCl}_{3}\right)$ (taken as a mixture of diastereomers): $\delta$ (major diastereomer) $1.26(\mathrm{~d}, J=6.9 \mathrm{~Hz}, 3 \mathrm{H}), 2.21(\mathrm{~s}, 3 \mathrm{H}), 3.71-3.77(\mathrm{~m}, 1 \mathrm{H}), 3.88-3.94(\mathrm{~m}$, $1 \mathrm{H}), 5.10-5.15(\mathrm{~m}, 2 \mathrm{H}), 5.92-6.04(\mathrm{~m}, 1 \mathrm{H}), 6.83-7.80(\mathrm{~m}, 10 \mathrm{H}) ; \delta$ (minor diastereomer ) $0.98(\mathrm{~d}$, $J=6.6 \mathrm{~Hz}, 3 \mathrm{H}), 4.88-4.97(\mathrm{~m}, 2 \mathrm{H}) ;{ }^{13} \mathrm{C} \mathrm{NMR}\left(75 \mathrm{MHz}, \mathrm{CDCl}_{3}\right): \delta$ (major diastereomer) 203.4, 139.2 , 132.7, 129.3, 129.1, 128.7, 128.4, 128.2, 128.0, 127.5, 116.4, 52.5, 45.2, 20.9, 16.5; MS (EI) m/z (rel) 249 (13), 159 (6), 131 (88), 115 (17), 105 (100), 91 (38), 77 (57); IR (film) 2977 (w), 2929 (w), 1683 (s), 1448 (m), 1208 (m), 973 (m), 708 (m); HRMS for $\mathrm{C}_{19} \mathrm{H}_{20} \mathrm{O}$ : 264.1514; Found: 264.1514; HPLC (Chiralcel OJ-H, hexane/i-Propanol $=90 / 10,0.7 \mathrm{~mL} / \mathrm{min}, 254 \mathrm{~nm}$ ) $\mathrm{t}_{\mathrm{R}}=$ 
$7.99 \mathrm{~min}, 8.86 \mathrm{~min}$.<smiles>C=C[C@H](C(=O)c1ccccc1)c1cccc(Br)c1</smiles>

\section{2-Methyl-1-phenyl-3-(m-bromo-phenyl)-pent-4-en-1-one (3d)}

Yield: 85\%; B/L: >98/2; anti:syn: 10:1; ee (anti-3d): 99\%.

${ }^{1} \mathrm{H}$ NMR $\left(300 \mathrm{MHz}, \mathrm{CDCl}_{3}\right.$ ) (taken as a mixture of diastereomers): $\delta$ (major diastereomer) 1.26 $(\mathrm{d}, J=6.6 \mathrm{~Hz}, 3 \mathrm{H}), 3.69-3.75(\mathrm{~m}, 1 \mathrm{H}), 3.86-3.91(\mathrm{~m}, 1 \mathrm{H}), 5.14(\mathrm{~d}, J=16.8 \mathrm{~Hz}, 1 \mathrm{H}), 5.15(\mathrm{~d}, J=$ $11.1 \mathrm{~Hz}, 1 \mathrm{H}), 5.89-6.01(\mathrm{~m}, 1 \mathrm{H}), 7.00-7.53(\mathrm{~m}, 7 \mathrm{H}), 7.79-7.82(\mathrm{~m}, 2 \mathrm{H}) ; \delta$ (minor diastereomer ) $0.99(\mathrm{~d}, J=6.6 \mathrm{~Hz}, 3 \mathrm{H}), 4.93-4.99(\mathrm{~m}, 2 \mathrm{H}) ;{ }^{13} \mathrm{C} \mathrm{NMR}\left(75 \mathrm{MHz}, \mathrm{CDCl}_{3}\right): \delta$ (major diastereomer) 203.0, 145.2, 138.3, 132.9, 130.8, 130.0, 129.4, 128.6, 128.2, 128.0, 126.3, 122.5, 117.3, 52.7, 45.0, 16.6; MS (EI) 328 (M+, 1), 313 (4), 195 (2), 129 (6), 116 (32), 105 (100), 77 (41); HRMS for $\mathrm{C}_{18} \mathrm{H}_{17} \mathrm{OBr}$ : 328.0463; Found: 328.0464. HPLC (Chiralcel OJ-H, hexane/i-Propanol = 90/10, $0.7 \mathrm{~mL} / \mathrm{min}, 254 \mathrm{~nm}) \mathrm{t}_{\mathrm{R}}=7.29 \mathrm{~min}, 7.93 \mathrm{~min}$.<smiles>C=C[C@H](c1ccc(OC)cc1)[C@@H](C)C(=O)c1ccccc1</smiles>

\section{2-Methyl-1-phenyl-3-(p-methoxy-phenyl)-pent-4-en-1-one (3e)}

Yield: 73\%; B/L: >98/2; anti:syn: 7:1; ee (anti-3e): 92\%.

$[\alpha]_{\mathrm{D}}{ }^{25}=-46.3^{\circ}\left(1.56, \mathrm{CHCl}_{3}\right) ;{ }^{1} \mathrm{H} \mathrm{NMR}\left(300 \mathrm{MHz}, \mathrm{CDCl}_{3}\right)$ (taken as a mixture of diastereomers): $\delta$ (major diastereomer) $1.25(\mathrm{~d}, J=6.6 \mathrm{~Hz}, 3 \mathrm{H}), 3.69-3.91(\mathrm{~m}, 5 \mathrm{H}), 5.10(\mathrm{~d}, J=12.0 \mathrm{~Hz}, 1 \mathrm{H}), 5.11$ $(\mathrm{d}, J=15.3 \mathrm{~Hz}, 1 \mathrm{H}), 5.93-6.05(\mathrm{~m}, 1 \mathrm{H}), 6.72(\mathrm{~d}, J=9.0 \mathrm{~Hz}, 2 \mathrm{H}), 7.11(\mathrm{~d}, J=8.7 \mathrm{~Hz}, 2 \mathrm{H})$, 7.36-7.52 (m, 3H), $7.80(\mathrm{~d}, J=7.5 \mathrm{~Hz}, 2 \mathrm{H}) ; \delta$ (minor diastereomer ) $0.98(\mathrm{~d}, J=6.6 \mathrm{~Hz}, 3 \mathrm{H})$, 4.88-4.97 (m, 2H); ${ }^{13} \mathrm{C}$ NMR (75 MHz, $\mathrm{CDCl}_{3}$ ): $\delta$ (major diastereomer) 203.6, 139.2, 132.7, 129.3, 128.7, 128.6, 128.4, 128.2, 128.0, 116.2, 113.8, 55.1, 52.1, 45.3, 16.5; MS (EI) m/z (rel) 280 (M+, 2), 265 (16), 147 (83), 115 (24), 105 (100), 91 (49), 77 (96), 51 (39); IR (film) 1677 (s), 1511 (s), 1247 (m), 1178 (m), 1031 (m), 974 (m), 828 (m), 707 (m); HRMS for $\mathrm{C}_{19} \mathrm{H}_{20} \mathrm{O}_{2}$ : 
280.1463; Found: 280.1459; HPLC (Chiralcel OJ-H, hexane/i-Propanol $=90 / 10,0.7 \mathrm{~mL} / \mathrm{min}$, $254 \mathrm{~nm}) \mathrm{t}_{\mathrm{R}}=13.51 \mathrm{~min}, 18.74 \mathrm{~min}$.<smiles>C=CC(C(=O)c1ccccc1)[C@H](C)c1cccc2ccccc12</smiles>

\section{2-Methyl-1-phenyl-3-(1-naphthyl)-pent-4-en-1-one (3f)}

Yield: 83\%; B/L: >98/2; anti:syn: 20:1; ee (anti-3f): 99\%.

$[\alpha]_{\mathrm{D}}{ }^{25}=-114.2^{\circ}\left(1.23, \mathrm{CHCl}_{3}\right) ;{ }^{1} \mathrm{H}$ NMR $\left(300 \mathrm{MHz}, \mathrm{CDCl}_{3}\right)$ (taken as a mixture of diastereomers): $\delta$ (major diastereomer) $1.40(\mathrm{~d}, J=6.9 \mathrm{~Hz}, 3 \mathrm{H}), 4.19-4.24(\mathrm{~m}, 1 \mathrm{H}), 4.64(\mathrm{dd}, J=$ $9.6,9.6 \mathrm{~Hz}, 1 \mathrm{H}), 5.15(\mathrm{~d}, J=10.5 \mathrm{~Hz}, 1 \mathrm{H}), 5.19$ (d, $J=16.8 \mathrm{~Hz}, 1 \mathrm{H}), 6.03-6.15(\mathrm{~m}, 1 \mathrm{H}), 7.24-7.63$ $(\mathrm{m}, 8 \mathrm{H}), 7.77-7.82(\mathrm{~m}, 3 \mathrm{H}), 8.30(\mathrm{~d}, J=8.7 \mathrm{~Hz}, 1 \mathrm{H}) ; \delta$ (minor diastereomer ) $0.99(\mathrm{~d}, J=6.9 \mathrm{~Hz}$, $3 \mathrm{H}), 4.91-4.98(\mathrm{~m}, 2 \mathrm{H}) ;{ }^{13} \mathrm{C} \mathrm{NMR}\left(75 \mathrm{MHz}, \mathrm{CDCl}_{3}\right): \delta$ (major diastereomer) 203.3, 139.2, 139.1, 136.9, 134.0, 132.7, 131.4, 128.8, 128.5, 128.0, 127.0, 126.0, 125.5, 125.1, 123.7, 123.6, 116.9, 47.1, 44.6, 17.0; MS (EI) m/z (rel) 300 (5), 285 (4), 195 (6), 167 (80), 165 (40), 152 (26), 105 (100), 77 (68), 51 (22); IR (film) 3059(w), 2976 (w), 1682 (s), 1597 (m), 1449 (m), 1255 (m), 1207 (m), 971 (s), 919 (m), 780 (s), 708 (m); HRMS for $\mathrm{C}_{22} \mathrm{H}_{20} \mathrm{O}$ : 300.1514; Found: 300.1516; HPLC (Chiralcel OJ-H, hexane/i-Propanol = 90/10, $0.5 \mathrm{~mL} / \mathrm{min}, 254 \mathrm{~nm}) \mathrm{t}_{\mathrm{R}}=18.12 \mathrm{~min}, 19.29$ $\min$.

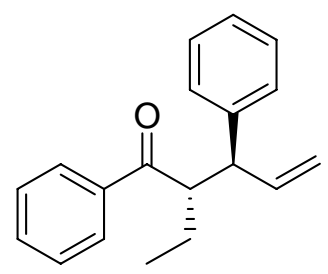

\section{2-Ethyl-1,3-diphenyl-pent-4-en-1-one (3g)}

Yield: 88\%; B/L: >98/2; anti:syn: 7:1; ee (anti-3g): 92\%.

$[\alpha]_{\mathrm{D}}{ }^{25}=-68.0^{\circ}\left(0.96, \mathrm{CHCl}_{3}\right) ;{ }^{1} \mathrm{H} \mathrm{NMR}\left(300 \mathrm{MHz}, \mathrm{CDCl}_{3}\right)$ (taken as a mixture of diastereomers): $\delta$ (major diastereomer) $0.84(\mathrm{t}, J=7.5 \mathrm{~Hz}, 3 \mathrm{H}), 1.75-1.87(\mathrm{~m}, 2 \mathrm{H}), 3.71-3.77(\mathrm{~m}, 1 \mathrm{H}), 3.82-3.90$ (m, 1H), $5.12(\mathrm{~d}, J=9.9 \mathrm{~Hz}, 1 \mathrm{H}), 5.14(\mathrm{~d}, J=17.1 \mathrm{~Hz}, 1 \mathrm{H}), 5.98-6.10(\mathrm{~m}, 1 \mathrm{H}), 7.01-7.56(\mathrm{~m}, 8 \mathrm{H})$, $7.74(\mathrm{~d}, J=7.8 \mathrm{~Hz}, 2 \mathrm{H}) ; \delta$ (minor diastereomer ) $0.71(\mathrm{t}, J=7.5 \mathrm{~Hz}, 3 \mathrm{H}), 4.85-4.97(\mathrm{~m}, 2 \mathrm{H})$, 5.86-5.92 (m, $1 \mathrm{H}) ;{ }^{13} \mathrm{C} \mathrm{NMR}\left(75 \mathrm{MHz}, \mathrm{CDCl}_{3}\right): \delta$ (major diastereomer) 203.8, 139.2, 132.6, 
128.6, 128.4, 128.3, 128.2, 127.9, 127.7, 126.3, 116.4, 52.5, 52.1, 24.1, 11.4; MS (EI) m/z (rel) 264 (M+, 2), 235 (34), 117 (100), 105 (73), 91 (17), 77 (41); IR (film) 2964 (w), 1751 (s), 1683 (s), 1448 (m), 1265 (s), 926 (m), 701 (m); HRMS for $\mathrm{C}_{19} \mathrm{H}_{20} \mathrm{O}$ : 264.1514; Found: 264.1504. HPLC (Chiralcel OJ-H, hexane/i-Propanol $=90 / 10,0.7 \mathrm{~mL} / \mathrm{min}, 254 \mathrm{~nm}) \mathrm{t}_{\mathrm{R}}=9.43 \mathrm{~min}, 12.77$ min.<smiles>C=CC(c1ccccc1)C(C(=O)c1ccccc1)C(=O)c1ccccc1</smiles>

\section{2-Propyl-1,3-diphenyl-pent-4-en-1-one (3h)}

Yield: 88\%; B/L: >98/2; anti:syn: 8:1; ee (anti-3h): 98\%.

$[\alpha]_{\mathrm{D}}{ }^{25}=-47.7^{\circ}\left(1.12, \mathrm{CHCl}_{3}\right) ;{ }^{1} \mathrm{H} \mathrm{NMR}\left(300 \mathrm{MHz}, \mathrm{CDCl}_{3}\right)$ (taken as a mixture of diastereomers): $\delta$ (major diastereomer) $0.86(\mathrm{t}, J=7.2 \mathrm{~Hz}, 3 \mathrm{H}) 1.18-1.30(\mathrm{~m}, 2 \mathrm{H}), 1.72-1.84(\mathrm{~m}, 2 \mathrm{H}), 3.73(\mathrm{t}, J=$ $9.6 \mathrm{~Hz}, 1 \mathrm{H}), 3.91(\mathrm{dt}, J=9.6,3.6 \mathrm{~Hz}, 1 \mathrm{H}), 5.12-5.18(\mathrm{~m}, 2 \mathrm{H}), 6.00-6.13(\mathrm{~m}, 1 \mathrm{H}), 7.03-7.75(\mathrm{~m}$, $10 \mathrm{H}) ; \delta$ (minor diastereomer ) $0.71(\mathrm{t}, J=7.2 \mathrm{~Hz}, 3 \mathrm{H}), 4.85-4.98(\mathrm{~m}, 2 \mathrm{H}), 5.87-5.98(\mathrm{~m}, 1 \mathrm{H}) ;{ }^{13} \mathrm{C}$ NMR (75 MHz, $\mathrm{CDCl}_{3}$ ): $\delta$ (major diastereomer) 204.1, 142.4, 139.2, 132.5, 128.5, 128.3, 128.2, 127.8, 127.7, 126.2, 116.4, 53.1, 50.8, 33.6, 14.3, 20.5; MS (EI) m/z (rel) 278 (M+, 1), 235 (26), 117 (100), 105 (75), 91 (24), 77 (35); HRMS for $\mathrm{C}_{20} \mathrm{H}_{22} \mathrm{O}$ : 278.1671; Found: 278.1669. HPLC (Chiralcel OJ-H, hexane/i-Propanol = 90/10, $0.7 \mathrm{~mL} / \mathrm{min}, 254 \mathrm{~nm}$ ) $\mathrm{t}_{\mathrm{R}}=8.04 \mathrm{~min}, 10.56 \mathrm{~min}$.<smiles>C=C[C@H](C(=O)c1ccc(OC)cc1)[C@@H](C)c1ccccc1</smiles>

\section{2-Methyl-1-(p-methoxy-phenyl)-3-phenyl-pent-4-en-1-one (3i)}

Yield: 82\%; B/L: >98/2; anti:syn: 7:1; ee (anti-3i): 97\%.

$[\alpha]_{\mathrm{D}}{ }^{25}=-59.7^{\circ}\left(1.27, \mathrm{CHCl}_{3}\right) ;{ }^{1} \mathrm{H} \mathrm{NMR}\left(300 \mathrm{MHz}, \mathrm{CDCl}_{3}\right)$ (taken as a mixture of diastereomers): $\delta$ (major diastereomer) $1.25(\mathrm{~d}, J=6.6 \mathrm{~Hz}, 3 \mathrm{H}), 3.72-3.91(\mathrm{~m}, 5 \mathrm{H}), 5.12(\mathrm{~d}, J=9.6 \mathrm{~Hz}, 1 \mathrm{H}), 5.13$ (d, $J=18.0 \mathrm{~Hz}, 1 \mathrm{H}), 5.94-6.03(\mathrm{~m}, 1 \mathrm{H}), 6.84-7.27(\mathrm{~m}, 8 \mathrm{H}), 7.81(\mathrm{~d}, J=8.7 \mathrm{~Hz}, 2 \mathrm{H}) ; \delta$ (minor diastereomer ) $0.96(\mathrm{~d}, J=6.6 \mathrm{~Hz}, 3 \mathrm{H}), 4.89-4.97(\mathrm{~m}, 2 \mathrm{H}) ;{ }^{13} \mathrm{C} \mathrm{NMR}\left(75 \mathrm{MHz}, \mathrm{CDCl}_{3}\right): \delta$ (major 
diastereomer) 201.8, 163.2, 143.0, 139.2, 130.3, 130.2, 128.4, 127.6, 126.2, 116.5, 113.6, 55.4, 53.1, 44.5, 16.7; MS (EI) m/z (rel) 280 (M+, 2), 265 (5), 150 (8), 135 (100), 117 (38), 107 (8), 91 (10), 77 (20); IR (film) 2974 (w), 1675 (s), 1601 (s), 1257 (m), 1172 (s), 1031 (m), 701 (m); HRMS for $\mathrm{C}_{19} \mathrm{H}_{20} \mathrm{O}_{2}: 280.1463$; Found: 280.1469. HPLC (Chiralcel OJ-H, hexane/i-Propanol $=$ 90/10, $0.7 \mathrm{~mL} / \mathrm{min}, 254 \mathrm{~nm}$ ) $\mathrm{t}_{\mathrm{R}}=15.93 \mathrm{~min}, 17.72 \mathrm{~min}$.<smiles>C=CC(C(=O)c1cccc2ccccc12)C(C)c1ccccc1</smiles>

\section{2-Methyl-1-(1-naphthyl)-3-phenyl-pent-4-en-1-one (3j)}

Yield: 89\%; B/L: >98/2; anti:syn: 21:1; ee (anti-3j): 93\%.

$[\alpha]_{\mathrm{D}}{ }^{25}=49.4^{\mathrm{o}}\left(1.50, \mathrm{CHCl}_{3}\right) ;{ }^{1} \mathrm{H} \mathrm{NMR}\left(300 \mathrm{MHz}, \mathrm{CDCl}_{3}\right)$ (taken as a mixture of diastereomers): $\delta$ (major diastereomer) $1.35(\mathrm{~d}, J=6.3 \mathrm{~Hz}, 3 \mathrm{H}), 3.79-3.88(\mathrm{~m}, 2 \mathrm{H}), 5.16(\mathrm{dd}, J=15.3,11.4 \mathrm{~Hz}$, $2 \mathrm{H}), 6.00-6.12(\mathrm{~m}, 1 \mathrm{H}), 7.07-8.04(\mathrm{~m}, 12 \mathrm{H}) ; \delta$ (minor diastereomer $) 1.08(\mathrm{~d}, J=5.7 \mathrm{~Hz}, 3 \mathrm{H})$, 4.93-5.02 (m, 2H); MS (EI) m/z (rel) 184 (23), 155 (100), 127 (84), 77 (15); IR (film) 2979 (w), 1683 (s), 1508 (m), 1230 (m), $1110(\mathrm{~m}), 797$ (m), $776(\mathrm{~m}), 732(\mathrm{~m})$; HRMS for $\mathrm{C}_{22} \mathrm{H}_{20} \mathrm{O}$ : 300.1514; Found: 300.1514. HPLC (Chiralcel OJ-H, hexane/i-Propanol $=90 / 10,0.5 \mathrm{~mL} / \mathrm{min}$, $254 \mathrm{~nm}) \mathrm{t}_{\mathrm{R}}=19.34 \mathrm{~min}, 22.02 \mathrm{~min}$.<smiles>C=C[C@H](C(=O)C1CCCCC1)c1ccc(OC)cc1</smiles>

\section{1-Cyclohexyl-3-(4'-methoxyphenyl)-2-methylpent-4-en-1-one (3k)}

Reaction condition: Molar ratio of $\mathbf{1} /\left[\mathrm{Pd}\left(\mathrm{C}_{3} \mathrm{H}_{5}\right) \mathrm{Cl}\right]_{2} /\left(R_{\text {phos }}, \quad R\right)-\mathbf{L 6} / \mathrm{LiHMDS} / \mathrm{LiCl} / \mathbf{2}=$ 100/5/10/120/200/120

Yield: 72\%; B/L: >98/2; anti:syn: 5:1; ee (anti-3k): 99\%.

$[\alpha]_{\mathrm{D}}{ }^{25}=-25.5^{\circ}\left(1.07, \mathrm{CHCl}_{3}\right) ;{ }^{1} \mathrm{H}$ NMR $\left(300 \mathrm{MHz}, \mathrm{CDCl}_{3}\right): \delta$ (major diastereomer) $0.80-1.30(\mathrm{~m}$, $10 \mathrm{H}), 1.55-2.03(\mathrm{~m}, 7 \mathrm{H}), 2.98-3.04(\mathrm{~m}, 1 \mathrm{H}), 3.38-3.45(\mathrm{~m}, 1 \mathrm{H}), 3.76(\mathrm{~s}, 3 \mathrm{H}), 5.05-5.11(\mathrm{~m}, 2 \mathrm{H})$, $5.86-5.95(\mathrm{~m}, 1 \mathrm{H}), 6.79(\mathrm{~d}, J=8.7 \mathrm{~Hz}, 2 \mathrm{H}), 7.07(\mathrm{~d}, J=8.7 \mathrm{~Hz}, 2 \mathrm{H}) ; \delta$ (minor diastereomer ) 
4.89-4.95 (2H); ${ }^{13} \mathrm{C}$ NMR (75 MHz, $\left.\mathrm{CDCl}_{3}\right): \delta$ (major diastereomer) 216.9, 157.9, 139.2, 135.0, 128.8, 116.0, 113.8, 55.1, 52.4, 51.3, 49.7, 28.2, 25.6, 25.3, 16.3; MS (EI) m/z (rel) $286(\mathrm{M}+, 2)$, 271 (8), 203 (25), 147 (100), 91 (14), 83 (29), 55 (14), 41 (10); IR (film) 2932 (s), 1706 (s), 1512 (s), 1248 (s), 1179 (m), 1037 (m), 992 (m), 833 (m); HRMS for $\mathrm{C}_{19} \mathrm{H}_{26} \mathrm{O}_{2}$ : 286.1933; Found: 286.1935; elemental analysis: calcd (\%) for $\mathrm{C}_{19} \mathrm{H}_{26} \mathrm{O}_{2}$ : C 79.68, H 9.15; found: C 55, H 9.14. HPLC (Chiralcel OJ-H, hexane/i-Propanol $=85 / 15,0.6 \mathrm{~mL} / \mathrm{min}, 230 \mathrm{~nm}) \mathrm{t}_{\mathrm{R}}=7.42 \mathrm{~min}, 8.88$ $\min$.

\section{Procedure for reduction:}

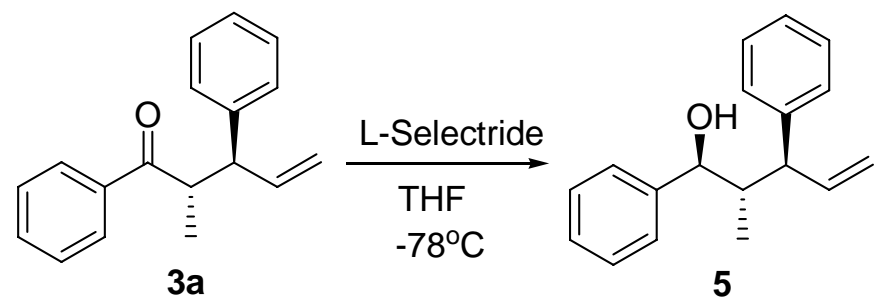

3a (30 mg, $0.12 \mathrm{mmol})$ was dissolved in freshly distilled THF $(1.5 \mathrm{~mL})$ under Argon and cooled to $-78^{\circ} \mathrm{C}$. At this temperature, L-Selectride $(0.25 \mathrm{ml}, 0.25 \mathrm{mmol}, 1.0 \mathrm{M}$ in THF $)$ was added dropwise, and the resulting mixture was stirred for 2 hours. Then acetone $(0.25 \mathrm{~mL})$ was added, followed by $\mathrm{H}_{2} \mathrm{O}_{2}(0.5 \mathrm{~mL}, 30 \%)$. The reaction mixture was warmed to room temperature continually stirred for another 1 hour. $\mathrm{H}_{2} \mathrm{O}(3 \mathrm{~mL})$ and washed with ether $(10 \mathrm{~mL} \times 3)$. The organic layer were combined and dried over $\mathrm{Na}_{2} \mathrm{SO}_{4}$. The solvent was removed under reduced pressure, and the residue was purified by flash column chromatography on silica gel with petroleum/ethyl acetate $(5 / 1)$ as eluent to give $5(100 \%)$ as a white solid:

\section{(1S, 2S, 3S)-2-Methyl-1,3-diphenyl-pent-4-en-1-ol (5)}

${ }^{1} \mathrm{H}$ NMR $\left(300 \mathrm{MHz}, \mathrm{CDCl}_{3}\right): \delta 0.65(\mathrm{~d}, J=7.2 \mathrm{~Hz}, 3 \mathrm{H}), 1.96(\mathrm{br}, 1 \mathrm{H}), 2.28-2.35(\mathrm{~m}, 1 \mathrm{H}), 3.78$ $(\mathrm{dd}, J=9.6,5.4 \mathrm{~Hz}, 1 \mathrm{H}), 4.48(\mathrm{~d}, J=7.8 \mathrm{~Hz}, 1 \mathrm{H}), 5.17-5.24(\mathrm{~m}, 2 \mathrm{H}), 6.11-6.23(\mathrm{~m}, 1 \mathrm{H}), 7.21-7.37$ (m, 10H); ${ }^{13} \mathrm{C}$ NMR $\left(75 \mathrm{MHz}, \mathrm{CDCl}_{3}\right): \delta 143.9,143.0,137.7,128.4,128.2,127.9,127.6,127.0$, 126.0, 117.1, 76.6, 50.4, 45.0, 11.6; MS (EI) m/z (rel) 252 (M+, 1), 234 (6), 117 (100), 107 (93), 91 (59), 79 (94), 77 (81), 57 (30); IR (KBr) 3361 (s), 3025 (w), 2909 (w), 1491 (m), 1452 (m), 1015 (m), $900(\mathrm{~m}), 760(\mathrm{~m}), 706(\mathrm{~s}), 521(\mathrm{~m})$; HRMS for $\mathrm{C}_{18} \mathrm{H}_{18}\left(\mathrm{M}-\mathrm{H}_{2} \mathrm{O}\right)^{+}:$234.1409. Found: 234.1407. 


\section{Procedure for reaction of alcohol 5 with $(S)$-Naproxen acid chloride:}<smiles>C=C[C@H](c1ccccc1)[C@H](C)[C@H](O)c1ccccc1</smiles><smiles>C=C[C@H](c1ccccc1)[C@H](C)[C@H](OC(=O)[C@H](C)c1ccccc1)c1ccc2cc(OC)ccc2c1</smiles>

5

$5(25.2 \mathrm{mg}, 0.1 \mathrm{mmol})$ and $\mathrm{CH}_{2} \mathrm{Cl}_{2}(2 \mathrm{~mL})$ were added to a dry Schlenk tube under Argon. Pyridine $(0.3 \mathrm{~mL})$ and $(S)$-Naproxen acid chloride $(40.0 \mathrm{mg}, 0.15 \mathrm{mmol})$ were added, and the mixture was stirred at room temperature. After the reaction was complete, $\mathrm{H}_{2} \mathrm{O}(5 \mathrm{~mL})$ and $\mathrm{CH}_{2} \mathrm{Cl}_{2}(5 \mathrm{~mL})$ were added and separated. The aqueous was washed with $\mathrm{CH}_{2} \mathrm{Cl}_{2}(3 \times 10 \mathrm{~mL})$ and the organic layers were combined, dried over $\mathrm{Na}_{2} \mathrm{SO}_{4}$. The solvent was removed under reduced pressure and the residue was purified by flash column chromatography on silica gel with petroleum/ethyl acetate (10/1) as eluent to give $6(90 \%)$ as a white solid: ${ }^{1} \mathrm{H}$ NMR (300 MHz, $\left.\mathrm{CDCl}_{3}\right): \delta 0.59(\mathrm{~d}, J=7.2 \mathrm{~Hz}, 3 \mathrm{H}), 1.54(\mathrm{~d}, J=7.2 \mathrm{~Hz}, 3 \mathrm{H}), 2.31-2.38(\mathrm{~m}, 1 \mathrm{H}), 3.43(\mathrm{dd}, J=9.6$, $5.4 \mathrm{~Hz}, 1 \mathrm{H}), 3.84(\mathrm{q}, J=6.9 \mathrm{~Hz}, 1 \mathrm{H}), 3.92(\mathrm{~s}, 3 \mathrm{H}), 4.91(\mathrm{~d}, J=17.1 \mathrm{~Hz}, 1 \mathrm{H}), 5.11(\mathrm{~d}, J=9.6 \mathrm{~Hz}$, $1 \mathrm{H}), 5.47(\mathrm{~d}, J=8.7 \mathrm{~Hz}, 1 \mathrm{H}), 6.02(\mathrm{dt}, J=17.1,9.9 \mathrm{~Hz}, 1 \mathrm{H}), 7.01-7.32(\mathrm{~m}, 14 \mathrm{H}), 7.50(\mathrm{~s}, 1 \mathrm{H})$, $7.64(\mathrm{dd}, J=15.0,8.4 \mathrm{~Hz}, 2 \mathrm{H})$; $\mathrm{MS}(\mathrm{ESI}) 487\left(\mathrm{M}+\mathrm{Na}^{+}\right), 519\left(\mathrm{M}+\mathrm{MeOH}+\mathrm{Na}^{+}\right)$.

ORTEP diagram of X-ray diffraction structure of $\mathbf{6}$

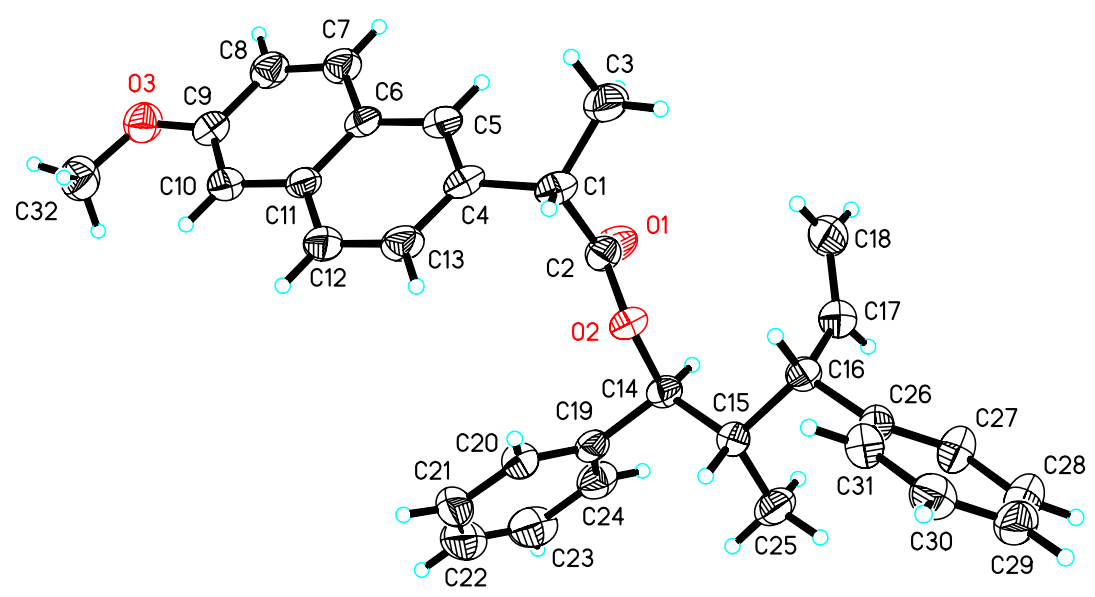

